\title{
Raman and infrared studies of cupric oxide
}

\author{
SOUMYENDU GUHA*, DALE PEEBLES and \\ J TERENCE WIETING \\ U.S. Naval Research Laboratory, Code 4652, Washington D.C. 20375-5000, USA
}

\begin{abstract}
Polarized Raman and fourier-transform infrared (FTIR) measurements have been made on a single crystal of $\mathrm{CuO}$. Group theory predicts nine vibrations of which three $\left(A_{g}, 2 B_{q}\right)$ are Raman-active and six $\left(3 A_{u}, 3 B_{u}\right)$ are infrared-active. We have observed three Raman modes at $296\left(A_{g}\right), 346\left(B_{g}^{1}\right)$ and $636\left(B_{g}^{2}\right) \mathrm{cm}^{-1}$. We have also observed six infrared modes at $146\left(B_{u}^{3}\right), 164\left(A_{u}^{2}\right), 355\left(A_{u}^{3}\right), 480\left(B_{u}^{1}\right), 542(?)$ and $603\left(B_{u}^{2}\right) \mathrm{cm}^{-1}$. The normal frequencies and eigenvectors have been calculated using Wilson's FG method; a good fit between theory and experiment has been obtained.
\end{abstract}

Keywords. Raman; infrared; cupric oxide.

\section{Introduction}

Cupric oxide is a basic ingredient in the preparation of high temperature superconductors; therefore, the characterization of its magnetic and vibrational properties is important. Although the magnetic properties of $\mathrm{CuO}$ have been investigated recently (Forsyth et al 1988; Yang et al 1989), Raman and infrared measurements on a cupric oxide single crystal have been reported only recently (Hanuza et al 1989; Goldstein et al 1990; Irwin et al 1990).

We present in this paper a summary of our polarized infrared and Raman measurements on a $\mathrm{CuO}$ single crystal.

\subsection{Crystal structure}

The crystal structure of $\mathrm{CuO}$ is monoclinic with four $\mathrm{CuO}$ units in the unit cell. The monoclinic unit cell belongs to the space group $C_{2 h}^{6}(C 2 / c)$ (Asbrink and Noorby 1970). The primitive cell contains two $\mathrm{CuO}$ units with the long direction parallel to the crystallographic $c$-axis. The long direction of the as-grown sample has been identified as the crystallographic $c$-axis, and the crystal planes are the (110) planes with angles of $72^{\circ}$ or $108^{\circ}$ between adjacent planes.

The crystal is biaxial-the unique $b(Y)$ axis is the bisector of the $108^{\circ}$ dihedral angle. The other two mutually perpendicular optic axes $(X, Z)$ were found to lie in the ac plane of the crystal.

Four samples of various sizes were studied ${ }^{\dagger}$. One of the samples was cut and polished to obtain a (001) face. Experiments conducted on the (001) face allowed us to distinguish between the $A_{g, u}$ and $B_{g, u}$ modes.

\footnotetext{
* The author is associated with SFA Inc., Landover, Maryland, USA

${ }^{\dagger} \mathrm{CuO}$ single crystals were obtained from two sources: (i) Superconics, St. Paul, MN, USA and (ii) Dr Terrell Vanderah, Naval Weapon Center, China Lake, CA, USA.
} 


\subsection{Normal modes and selection rules}

The primitive cell contains four atoms and hence there are twelve possible normal modes. With the help of a correlation diagram, the irreducible representations associated with the lattice vibrations of the primitive cell have been determined as $\Gamma=4 A_{u}+5 B_{u}+A_{g}+2 B_{g}$. There are three acoustic modes $\left(A_{u}+2 B_{u}\right)$ and six optic modes. The even modes are Raman-active, while the odd modes are infrared-active.

\section{Experiments}

Polarized Raman experiments were conducted in back scattering and in $90^{\circ}$ geometry. In these experiments the scattered light was collected, digitized and analysed by a Spex 1403 monochromator, a cooled detector and Spex DM 3000 software. The laser power was kept at $50 \mathrm{~mW}$, and the spectrometer resolution was $5 \mathrm{~cm}^{-1}$.

Polarized reflectance data were obtained with a fourier-transform infrared (FTIR) spectrophotometer (Bomem DA 3.26 model) in a near-reflection geometry. A globar source, a liquid helium-cooled-bolometer and a Mylar beam splitter were used to record the FTIR data. The data were analysed with a PDP 11-computer.

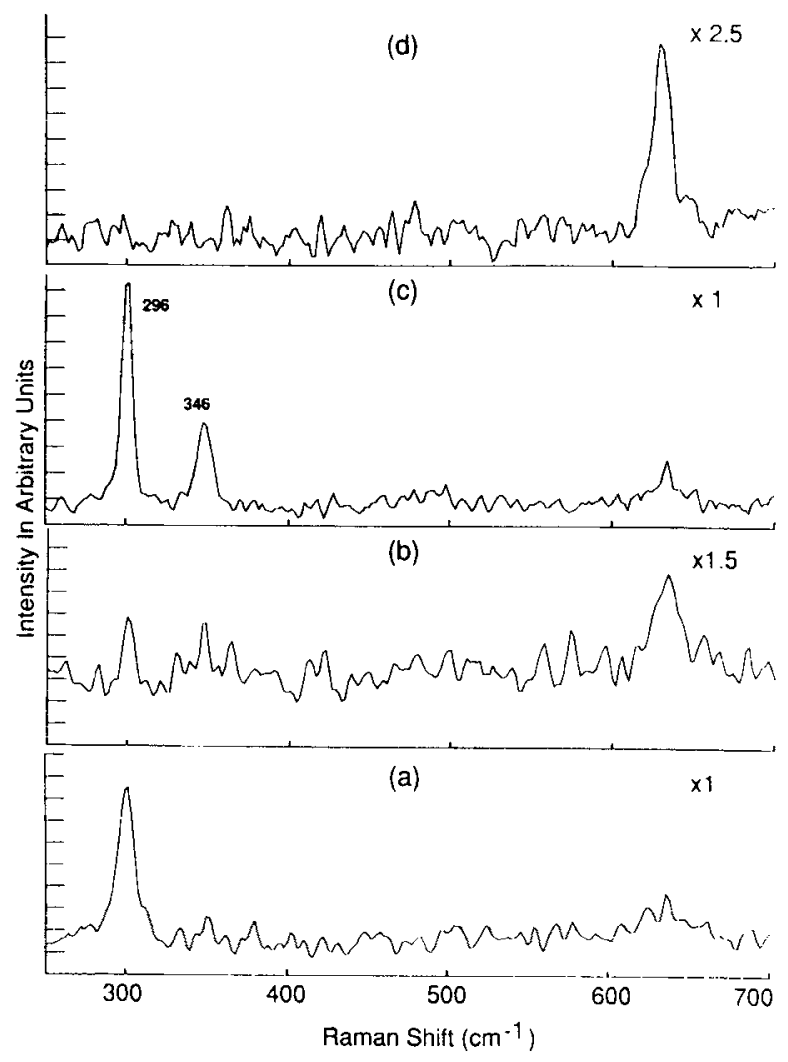

Figure 1. Raman spectra at room temperature: (a) the back-scattered $Y Y$ polarization spectrum from a (001) face; (b) the back-scattered $Y Z$ polarization spectrum from a $(001)$ face; (c) the $90^{\circ}$-geometry $(Y+Z, Y+Z)$ spectrum from an as-grown sample; (d) the $90^{\circ}$ geometry $Y Z$ spectrum from an as-grown sample. 


\subsection{Raman spectra}

Figure 1 shows the Raman spectra taken at room temperature. The back-scattered Raman spectra from the (001) face is shown by figures 1 (a) and (b). The $296 \mathrm{~cm}^{-1}$ peak in the $Y Y$ geometry is assigned to the $A_{g}$ mode, while the $636 \mathrm{~cm}^{-1}$ peak in the $(Y+Z, Y+Z)$ geometry is assigned to the $B_{g}$ mode. Figures $1(\mathrm{c})$ and (d) are the $90^{\circ}$ geometry Raman spectra from an as-grown sample. In this sample geometry the $(Y+Z, Y+Z)$ spectrum exhibits both the $A_{g}$ and $B_{g}$ modes as shown in figure 1(c), while the $Y Z$ spectrum shows only one of the $B_{g}$ components. This is probably due to the weak intensity of the other $\left(246 \mathrm{~cm}^{-1}\right) B_{g}$ component.

\subsection{Infrared spectra}

Figure 2(a) shows the reflectance data from an as-grown sample with two different polarizations of the incoming radiation. The polarizations are oriented along the two optic axes. The dashed and solid lines in figure 2 represent the $X$ and $Z$ polarizations of the incident radiation respectively. The real and imaginary parts of the dielectric function were obtained using a Kramers Kronig analysis. The longitudinal (LO) and
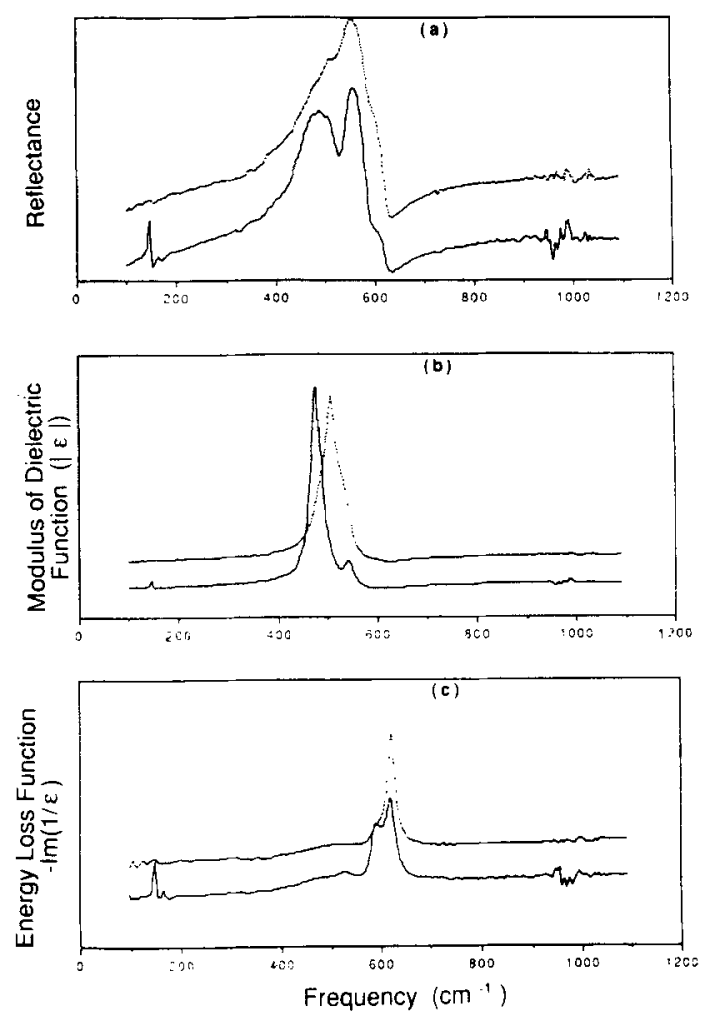

Figure 2 (a) Polarized infrared reflectance spectra from an as-grown sample. The two orientations of the incident polarizations are along the $X$ and $Z$ optic axes and are represented by the dashed and solid lines; (b) the TO peaks obtained from the modulus of the dielectric function; (c) the LO peaks obtained from the energy loss function. 
Table 1. Calculated and observed vibrational frequencies of CuO. The LO and TO frequencies are obtained from figures $2(b)$ and (c); the observed Raman frequencies are indicated by the letter $R$.

\begin{tabular}{cccccc}
\hline Mode type & $\begin{array}{c}\text { Calculated } \\
\left(\mathrm{cm}^{-1}\right)\end{array}$ & $\begin{array}{c}\text { Observed } \\
\left(\mathrm{cm}^{-1}\right)\end{array}$ & $\begin{array}{c}\text { Calculated } \\
\left(\mathrm{cm}^{-1}\right)\end{array}$ \\
\hline$A_{g}$ & 294 & 296 & $\mathrm{R}$ & & \\
$B_{g}^{1}$ & 348 & 346 & $\mathrm{R}$ & & \\
$B_{g}^{2}$ & 624 & 636 & $\mathrm{R}$ & & \\
$A_{u}^{1}$ & 421 & $?$ & & & \\
$A_{u}^{2}$ & 156 & 164 & TO & 165 & LO \\
$A_{u}^{3}$ & 365 & 355 & TO & & $?$ \\
$B_{u}^{1}$ & 474 & 480 & TO & 624 & LO \\
$B_{u}^{2}$ & 598 & 603 & TO & 593 & LO \\
$B_{u}^{3}$ & 146 & 145 & TO & 147 LO \\
\hline
\end{tabular}

transverse optic (TO) modes were obtained from the modulus of the dielectric function and the energy loss function $-\operatorname{Im}(1 /|\varepsilon|)$ which are shown in figures 2 (b) and (c). The frequencies for the LO and TO modes are listed in table 1.

\section{Normal mode analysis}

The observed Raman and IR frequencies are listed in table 1 . Normal mode frequencies were calculated using Wilson's F-G* method adapted to the crystal problem*. Five force constant parameters involving $\mathrm{Cu}-\mathrm{O}, \mathrm{Cu}-\mathrm{Cu}$ and $\mathrm{O}-\mathrm{O}$ stretches and $\mathrm{Cu}-\mathrm{O}-\mathrm{Cu}$ and $\mathrm{O}-\mathrm{Cu}-\mathrm{O}$ valence bendings were used to fit both Raman and IR data. The calculated frequencies are listed in table 1.

\section{Discussion and conclusion}

We have observed three Raman and six infrared modes which were identified as the $A_{g}, B_{g}$ and $A_{u}, B_{u}$ modes. Our assignments of the Raman frequencies to the $A_{g}$ $\left(296 \mathrm{~cm}^{-1}\right)$ and $B_{g}\left(346\right.$ and $\left.636 \mathrm{~cm}^{-1}\right)$ modes are complete and agree well with the normal mode calculation. However, the assignments of the infrared frequencies to $A_{u}$ and $B_{u}$ modes are only tentative at this point, since a more accurate determination of the frequencies as a function of the polarization of the incident radiation is required. This work is currently in progress.

\footnotetext{
*We recommend an excellent review of Wilson's F-G method extended to the crystal problem by George Turrell, Infrared and Raman spectra of crystals, p. 186, Academic Press, London and New York (1972).
} 


\section{References}

Asbrink S and Noorby L 1970 Acta Crystallogr. B26 8

Forsyth J B et al 1988 J. Phys. C21 2917

Goldstein H F et al 1990 Phys. Rev. B41 7192

Hagemann et al 1990 Solid State Commun 73447

Hanuza et al 1989 J. Mol. Struc. 19357

Irwin J C et al 1990 Physica C166 456

Yang B X et al 1989 Phys. Rev. B39 4343 